\title{
The Effect of eHealth Interventions on Physical Activity in Patients with Chronic Obstructive Pulmonary Disease: A Mini Review
}

\author{
Yoshiki Tsuchida'"\#, Sigrid N.W. Vorrink ${ }^{2,3 *}$ \\ 'Department of Physical Therapy, Nagoya University Graduate School of Medicine, Nagoya, Japan \\ ${ }^{2}$ Utrecht University of Applied Sciences, Research Group Technology for Healthcare Innovations, Utrecht, Netherlands \\ ${ }^{3}$ JointResearch, Department of Orthopaedic Surgery, OLVG Amsterdam, Netherlands
}

Article Info

\section{Article Notes}

Received: April 1, 2019

Accepted: May 28, 2019

\section{${ }^{*}$ Correspondence:}

*Dr. Sigrid N.W. Vorrink, PhD, Utrecht University of Applied Sciences, Research Group Technology for Healthcare Innovations, Utrecht, and JointResearch, Department of Orthopaedic Surgery, OLVG Amsterdam, Netherlands; Email: s.vorrink@olvg.nl.

"Mr. Yoshiki Tsuchida, PT, Department of Physical Therapy, Nagoya University Graduate School of Medicine, Nagoya, Japan; Email: yoshiki.tsuchida1228@gmail.com.

C 2019 Vorrink S, Tsuchida Y. This article is distributed under the terms of the Creative Commons Attribution 4.0 International License.

\section{Keywords}

eHealth

Physical activity

Chronic Obstructive Pulmonary Disease (COPD)
Abstract

Physical activity (PA) is important for patients with chronic obstructive pulmonary disease (COPD). eHealth interventions may have the possibility to increase and maintain PA. The objective of this mini review is to provide an overview of research published from 2016 to 2018 about the effect of eHealth on PA in patients with COPD.

Recent studies used phone calls, websites combined with monitoring devices and smartphone applications as eHealth interventions. These studies showed both positive and no effects of eHealth interventions on PA in patients with COPD. The effects seem to depend on the type of eHealth intervention. A multicomponent eHealth intervention including reinforcement, self-management, monitoring and exercise with phone calls, websites and pedometers may be useful for stimulating PA for short-term periods.

\section{Introduction}

Physical activity (PA) is important for patients with chronic obstructive pulmonary disease (COPD). A decrement of PA is associated with an increase in risk of mortality ${ }^{1}$. In addition, lower PA is associated with exacerbation ${ }^{2}$, hospitalization ${ }^{3}$ and all-cause mortality ${ }^{4}$. For example, an increase of physical activity after a COPD hospitalization contributes to a $76 \%$ reduction in rehospitalization ${ }^{5}$. In addition, a high PA level is associated with a lower risk of death in COPD patients (hazard ratio: $0.46,95 \%$ confidential interval: 0.33-0.64) ${ }^{4}$. Watz et al. (2014) reported in their systematic review that pulmonary rehabilitation (PR) had no unified effect on PA in patients with COPD in 10 studies. However, behavioral interventions had a positive effect in 11 studies $^{6}$. Mantoani et al. (2016) claimed that there were both positive and negative effects of PR on PA among 20 studies. They also mentioned that long-lasting PR and counseling seemed to increase PA in patients with $\mathrm{COPD}^{7}$.

eHealth interventions may have the possibility to increase and maintain PA. The definition of eHealth includes not only health service and information through the internet or related technology, but also a state of mind and a way of thinking ${ }^{8}$. It has been reported that eHealth interventions have contributed to a reduction in health care costs $^{9}$, maintain motivation for behavioral change ${ }^{10}$, improve both physical and clinical function ${ }^{11}$, and stimulate PA of older people with chronic conditions ${ }^{12}$. In 2015, a systematic review and metaanalysis including 9 studies showed the positive effects of eHealth on $\mathrm{PA}$ in patients with $\mathrm{COPD}^{13}$. It is possible that eHealth interventions 
can enhance adherence of PR and make it easier for patients to access a behavioral intervention. However, there have not been a lot of studies which investigated the effects of an eHealth intervention on PA in patients with COPD before 2015. In addition, no meta-analysis on this topic has been written since 2016 and it would be good to update this overview in the current mini review.

The objective of this mini review is to provide an overview of research published from 2016 to 2018 about the effect of eHealth on PA in patients with COPD.

\section{Types of eHealth Interventions}

In the systematic review published in 2015, 6 out of 9 studies used an eHealth intervention that consisted of regular phone calls, websites, mobile phone applications or monitoring devices such as a pedometer ${ }^{13}$. Sometimes they were combined with a different type of eHealth intervention, exercise intervention or education. These eHealth interventions mainly focused on reinforcement, feedback, giving information of symptoms and healthy habits, sending care plans and monitoring PA. Although this trend does not seem to change dramatically, a few new smart applications have been developed and tested after $2015^{14,15,16,17}$. Table 1 shows an overview of the types of eHealth interventions that are discussed in this mini review.

\section{Table1. Types of eHealth interventions}

\begin{tabular}{|l|l|}
\hline \multicolumn{1}{|c|}{ Types } & \multicolumn{1}{c|}{ Main aim } \\
\hline Phone call & reinforcement, feedback and giving information \\
\hline Website & $\begin{array}{l}\text { feedback, giving information, sending care } \\
\text { plans and monitoring }\end{array}$ \\
\hline $\begin{array}{l}\text { Smartphone } \\
\text { application }\end{array}$ & $\begin{array}{l}\text { reinforcement, feedback, sending care plans } \\
\text { and monitoring }\end{array}$ \\
\hline Monitoring devices & feedback and monitoring \\
\hline
\end{tabular}

\section{Effects of eHealth Intervention}

\section{Phone calls}

No studies using only phone calls have been published since 2016, but some studies combined phone calls with home-based exercise or action plans. Frank et al. (2016) used phone calls as a means of reinforcement toward home ergometer exercise with a remote monitoring system equipped in an ergometer in a crossover randomized study ${ }^{18}$. During the 3 months intervention phase, participants were called once a week only when they did not achieve their daily cycle time of 20 minutes. During the control phase, they were asked to perform the same exercise without phone calls. As a result, there was no significant difference of leisure time PA between the intervention and control phase, but daily training time was higher during the intervention phase.

Cameron-Tucker et al. (2016) also used phone calls to support health behavior including a home-based walking plan ${ }^{19}$. The intervention group was contacted via phone by community nurses two times a week during 8 to 12 weeks while the control group received usual care without phone calls. They reported no significant difference of self-reported walking time between either groups or time-points. Both studies had a relatively short intervention duration (3 months or less). Although a short-term intervention with phone calls may have the potential to maintain adherence of home-based exercise or action plans, it does not seem to be enough to enhance PA.

On the other hand, Bernocchi et al. (2018) reported a positive effect of phone calls on PA. This study consisted of a multicomponent intervention for 4 months (including phone calls, exercise and a behavioral intervention $)^{20}$. They provided patients who had both COPD and heart failure with advice about healthy habits, PA goals and exercise plans through regular phone calls. At 2 months after the intervention, PA measured by a questionnaire was improved as compared with usual outpatient care.

\section{Websites combined with monitoring devices}

Websites have often been used in combination with monitoring devices for PA. Wan et al. (2017) compared daily step count during 3 months between a website with pedometer group and pedometer only group ${ }^{21}$. The aim of the website was to support participants in their selfmanagement. It provided participants with individual PA goals and history of daily step counts. The website with pedometer group had a significantly higher increase in daily step count than the pedometer only group at the end of the intervention. However, they also reported this effect was not maintained after 12 months ${ }^{22}$.

When it comes to monitoring by a pedometer, the positive effect on PA in patients with COPD has been reported before $2016^{23,24}$. Recently, Tomasic et al. (2018) also claimed in their review article that monitoring by a pedometer could increase and maintain the level of $\mathrm{PA}^{25}$. On the other hand, there were few studies which reported the positive effects of monitoring on PA with other devices such as video-monitoring. Tsai et al. (2017) included patients for home-based group exercise and monitoring for 3 months through videoconference on a website ${ }^{26}$. They reported no effects of the intervention on PA although functional capacity and self-efficacy improved as compared with the control group which received usual care.

Hoaas et al. (2016) conducted a pilot study which had no control group to investigate the adherence of a longterm self-exercise program in 10 patients with moderate to severe $\mathrm{COPD}^{27}$. The intervention included a homebased treadmill exercise, a weekly videoconference with a physiotherapist by using a tablet computer and selfmanagement on a website. The website enabled patients to 
record daily symptoms, exercise duration and health states. In addition, physiotherapists could access this information and discuss it with the patients. They followed participants for 740 days on average and measured adherence and training frequency as an outcome of PA. As a result, they reported that the average training frequency was 1.7 sessions per week over the two-year follow up period. This corresponds to an average adherence rate of $56.2 \%$ to the training sessions, which they conclude is slightly higher than average. However, adherence rate did decline over the two years (69.1\% in year one and $40.5 \%$ in year two), which once again underscores the difficulty in maintaining PA results long term for COPD patients.

Websites with monitoring devices which assist patients in the self-management of PA have the possibility to stimulate daily PA in patients with COPD. The effect may not be maintained for long-term periods when the intervention is ceased, but websites with monitoring seem to maintain the motivation toward home-based exercise.

\section{Smartphone applications}

Some new smartphone applications have been developed since 2016. They are often used in combination with a website. Vorrink et al. (2016) investigated the effect of a smartphone app that stimulated PA through personalized goals, direct feedback and text messages from a physiotherapist ${ }^{14}$. The physiotherapist had insight into their patients' data via a website where they could adjust the PA goals and send group or individual messages. In this randomized controlled trial, the control group received usual care. The results showed no significant difference in PA between groups and time-points at 6 months (intervention period) and follow-up at 12 months.

Contrary to these results, Demeyer etal. (2017) reported positive effects of a smartphone app on PA in patients with $\mathrm{COPD}^{17}$. They implemented a 12-week coaching intervention using a smartphone app while the control group received usual care. The app showed patients their daily step counts and gave them PA goals and advice. At the end of the intervention period, the amount and intensity of PA significantly increased in the intervention group while PA decreased in the control group.

Bartlett et al. (2017) developed three prototypes of a smartphone app ${ }^{15}$. First, the "virtual coach system" assisted in setting a PA goal, gave advice and showed patients their activity data on the display of their smartphones. Secondly, the "music and maps system" enabled patients to see their PA goal, track their activity on the map and perform walking activities while listening to music. Thirdly, the "online community system" connected patients in order to share their PA, and stimulate competition and cooperation with each other. Although the changes in PA were not reported, they found through interviews and questionnaires that these apps were acceptable and likely to be persuasive for patients with COPD.

When it comes to smartphone apps, there was not enough evidence to draw conclusions. Further studies are necessary to discuss the effect of smartphone apps on PA in patients with COPD.

\section{Discussion}

Recent studies ${ }^{17,20,21,25,27}$ showed positive effects of eHealth on PA in patients with COPD while other studies $^{14,18,19,26}$ reported no effects. It seems to depend on the type, duration and frequency of the intervention. A multicomponent eHealth intervention including reinforcement, self-management, monitoring and exercise with phone calls, websites and pedometers may be useful for stimulating PA for short-term periods. Previous studies published before 2016 support this opinion. Altenburg et al. (2015) reported that a counseling program combined with the use of a pedometer had a positive effect on daily step count during 15 months for sedentary patients with COPD ${ }^{24}$. In addition, a previous systematic review including 26 studies showed that 12 or more calls over a 6-12 month period improved PA and health behavior ${ }^{28}$.

Although new smartphone apps have been developed, there were contradictor results among recent studies ${ }^{14,15,17}$. Lahham et al. (2016) reported that exercise training with counseling was more effective to stimulate PA than only exercise ${ }^{29}$. Williams et al. (2017) concluded that a counseling intervention for health behavior had a significant effect on smoking behavior, but not on PA in their systematic review ${ }^{30}$. Based on these studies (Lahham et al. and Williams et al.), it seems that eHealth interventions with smartphone apps need to be combined with exercise and counseling in order to successfully stimulate PA. However, there is not enough evidence to conclude anything. Future studies are needed to look into the effects of eHealth interventions using a smartphone app.

It has been reported that PA in patients with COPD is associated with physical and psychological factors. For example, Hayata et al. (2016) reported that a modified MRC (Medical Research Council breathlessness scale) grade $\geq 2$ could predict low levels of $\mathrm{PA}^{31}$. Dueñas-Espín et al. (2016) showed that depression symptoms were strongly associated with a reduction in $\mathrm{PA}^{32}$. In the systematic review, some physical and psychological factors, for example, hyperinflation, dyspnea, gas exchange and self-efficacy, were reported to have an association with $\mathrm{PA}^{33}$. Given these facts, it may be insufficient for some COPD patients to only use an eHealth intervention. It might be necessary to provide a patient who has serious physical and/or psychological problems with individual medical care including more than only an eHealth intervention. Future research is expected to make the effect of eHealth interventions clear. 
In conclusion, the current mini-review found from research published from 2016 to 2018 that certain types of eHealth intervention have the possibility to stimulate PA in patients with COPD. At this time, two randomized controlled trials are being conducted to compare the effects of online-based COPD rehabilitation on PA as compared with conventional outpatient rehabilitation ${ }^{34,35}$. Future research is still needed in order to find more effective eHealth interventions to stimulate PA in COPD patients. There should be a focus on maintaining the effects on PA with long term interventions.

\section{References}

1. Vaes AW, Garcia-Aymerich J, Marott JL, et al. Changes in physical activity and all-cause mortality in COPD. Eur Respir J. 2014; 44(5): 1199-1209.

2. Pitta F, Troosters T, Probst VS, et al. Physical Activity and Hospitalization for Exacerbation of COPD. Chest. 2006; 129(3): 536-544.

3. Esteban C, Arostegui I, Aburto M, et al. Influence of changes in physical activity on frequency of hospitalization in chronic obstructive pulmonary disease. Respirology. 2014; 19(3): 330-338.

4. Waschki B, Kirsten A, Holz O, et al. Physical activity is the strongest predictor of all-cause mortality in patients with COPD: a prospective cohort study. Chest. 2011; 140(2): 331-342.

5. Criner GJ, Bourbeau J, Diekemper RL, et al. Prevention of acute exacerbations of COPD: American College of Chest Physicians and Canadian Thoracic Society Guideline. Chest. 2015; 147(4): 894-942.

6. Watz H, Pitta F, Rochester CL, et al. An official European Respiratory Society statement on physical activity in COPD. Eur Respir J. 2014; 53(1).

7. Mantoani LC, Rubio N, McKinstry B, et al. Interventions to modify physical activity in patients with COPD: a systematic review. Eur Respir J. 2016; 48(1): 69-81.

8. Eysenbach G. What is e-health? J Med Internet Res. 2001; 3(2)

9. Polisena J, Coyle D, Coyle K, et al. Home telehealth for chronic disease management: A systematic review and an analysis of economic evaluations. Int J Technol Assess Health Care. 2009; 25(03): 339-349.

10. Walters JAE, Cameron-Tucker H, Courtney-Pratt H, et al. Supporting health behaviour change in chronic obstructive pulmonary disease with telephone health-mentoring: insights from a qualitative study. BMC Fam Pract. 2012; 13: 55.

11. Marquis N, Larivée P, Saey D, et al. In-Home Pulmonary Telerehabilitation for Patients with Chronic Obstructive Pulmonary Disease: A Pre-experimental Study on Effectiveness, Satisfaction, and Adherence. Telemed e-Health. 2015; 21(11): 870-879.

12. Posadzki P, Mastellos N, Ryan R, et al. Automated telephone communication systems for preventive healthcare and management of long-term conditions. Cochrane Database of Systematic Reviews. 2016; 12.
13. Lundell S, Holmner $\AA$, Rehn B, et al. Telehealthcare in COPD: A systematic review and meta-analysis on physical outcomes and dyspnea. Respir Med. 2015; 109(1): 11-26.

14. Vorrink SNW, Kort HSM, Troosters T, et al. Efficacy of an mHealth intervention to stimulate physical activity in COPD patients after pulmonary rehabilitation. Eur Respir J. 2016; 48(4): 1019-1029.

15. Bartlett YK, Webb TL, Hawley MS. Using Persuasive Technology to Increase Physical Activity in People With Chronic Obstructive Pulmonary Disease by Encouraging Regular Walking: A Mixed-Methods Study Exploring Opinions and Preferences. J Med Internet Res. 2017; 19(4).

16. Tey CK, An J, Chung WY. A Novel Remote Rehabilitation System with the Fusion of Noninvasive Wearable Device and Motion Sensing for Pulmonary Patients. Comput Math Methods Med. 2017; 2017: 5823740.

17. Demeyer H, Louvaris Z, Frei A, et al. Physical activity is increased by a 12-week semiautomated telecoaching programme in patients with COPD: a multicentre randomised controlled trial. Thorax. 2017; 72(5): 415-423.

18. Franke KJ, Domanski U, Schroeder M, et al. Telemonitoring of home exercise cycle training in patients with COPD. Int J Chron Obstruct Pulmon Dis. 2016; 11: 2821-2829.

19. Cameron-Tucker HL, Wood-Baker R, Joseph L, et al. A randomized controlled trial of telephone-mentoring with home-based walking preceding rehabilitation in COPD. Int J Chron Obstruct Pulmon Dis. 2016; 11: 1991-2000.

20. Bernocchi P, Vitacca M, La Rovere MT, et al. Home-based telerehabilitation in older patients with chronic obstructive pulmonary disease and heart failure: a randomised controlled trial. Age Ageing. 2018; 47(1): 82-88.

21. Wan ES, Kantorowski A, Homsy D, et al. Promoting physical activity in COPD: Insights from a randomized trial of a webbased intervention and pedometer use. Respir Med. 2017; 130: 102-110.

22. Moy ML, Martinez CH, Kadri R, et al. Long-Term Effects of an Internet-Mediated Pedometer-Based Walking Program for Chronic Obstructive Pulmonary Disease: Randomized Controlled Trial. J Med Internet Res. 2016; 18(8).

23. Mendoza L, Horta P, Espinoza J, et al. Pedometers to enhance physical activity in COPD: a randomised controlled trial. Eur Respir J. 2015; 45(2): 347-354.

24. Altenburg WA, Ten Hacken NHT, Bossenbroek L, et al. Shortand long-term effects of a physical activity counselling programme in COPD: A randomized controlled trial. Respir Med. 2015; 109: 112-121.

25. Tomasic I, Tomasic N, Trobec R, et al. Continuous remote monitoring of COPD patients-justification and explanation of the requirements and a survey of the available technologies. Med Biol Eng Comput. 2018; 56(4): 547-569.

26. Tsai LLY, McNamara RJ, Moddel C, et al. Home-based telerehabilitation via real-time videoconferencing improves endurance exercise capacity in patients with COPD: The randomized controlled TeleR Study. Respirology. 2017; 22(4): 699-707. 
27. Hoaas H, Andreassen HK, Lien LA, et al. Adherence and factors affecting satisfaction in long-term telerehabilitation for patients with chronic obstructive pulmonary disease: a mixed methods study. BMC Med Inform Decis Mak. 2016; 16: 26.

28. Eakin EG, Lawler SP, Vandelanotte C, et al. Telephone Interventions for Physical Activity and Dietary Behavior Change: A Systematic Review. Am J Prev Med. 2007; 2(5): 419434.

29. Lahham A, McDonald CF, Holland AE. Exercise training alone or with the addition of activity counseling improves physical activity levels in COPD: a systematic review and meta-analysis of randomized controlled trials. Int J Chron Obstruct Pulmon Dis. 2016; 11: 3121-3136.

30. Williams MT, Effing TW, Paquet C, et al. Counseling for health behavior change in people with COPD: systematic review. Int J Chron Obstruct Pulmon Dis. 2017; 12: 2165-2178.

31. Hayata A, Minakata Y, Matsunaga K, et al. Differences in physical activity according to mMRC grade in patients with COPD. Int J Chron Obstruct Pulmon Dis. 2016; 11: 2203-2208.

32. Dueñas-Espín I, Demeyer H, Gimeno-Santos E, et al. Depression symptoms reduce physical activity in COPD patients: a prospective multicenter study. Int J Chron Obstruct Pulmon Dis. 2016; 11(1): 1287.

33. Gimeno-Santos E, Frei A, Steurer-Stey C, et al. Determinants and outcomes of physical activity in patients with COPD: a systematic review. Thorax. 2014; 69(8): 731-739.

34. N Nyberg A, Wadell K, Lindgren $\mathrm{H}$, et al. Internet-based support for self-management strategies for people with COPD-protocol for a controlled pragmatic pilot trial of effectiveness and a process evaluation in primary healthcare. BMJ Open. 2017; 7(7).

35. Hansen H, Bieler T, Beyer N, et al. COPD online-rehabilitation versus conventional COPD rehabilitation - rationale and design for a multicenter randomized controlled trial study protocol (CORe trial). BMC Pulm Med. 2017; 17(1): 140. 